\title{
A Solution for a Water Quality Model in a Uniform Stream Channel Using New Iterative Method
}

\author{
M. Khalid \\ Department of Mathematical Sciences \\ Federal Urdu University of Arts, Sciences \& Techonology \\ University Road, Karachi-75300, Pakistan \\ Faheem Zaidi \\ Department of Mathematical Sciences \\ Federal Urdu University of Arts, Sciences \& Technology \\ University Road, Karachi-75300, Pakistan
}

\author{
Mariam Sultana \\ Department of Mathematical Sciences \\ Federal Urdu University of Arts, Sciences \& Technology \\ University Road, Karachi-75300, Pakistan \\ Javed Khan \\ Department of Mathematical Sciences \\ Federal Urdu University of Arts, Sciences \& Technology \\ University Road, Karachi-75300, Pakistan
}

\begin{abstract}
During the last decade, political awareness of river water quality issues has increased significantly. New environmental policies have a requirement for improved methods for the investigation and evaluation of river water quality. Moreover, the derivation and assessment of management practices is also necessary. An efficient Perturbation Iteration Algorithm for solving the water quality assessment model has been developed. The employed equation of this uniform flow model is one-dimensional Advection-DispersionReaction equation that has variable coefficients. This water quality model requires the calculation of the substance dispersion considering the water velocity in the channel. Numerical values are obtained by using the Runge-Kutta-Fehlberg fourth-fifth order method for comparison. It was discovered that Perturbation Iteration Algorithm solution gels well with the numerical solution. Two examples are included to demonstrate the efficiency, accuracy, and simplicity of the proposed method.
\end{abstract}

\section{Keywords:}

Water Quality Model; Uniform Channel; Advection-DispersionReaction Equation; Perturbation Iteration Algorithm; Semi Analytical Solution

\section{INTRODUCTION}

In recent times, the general public has become more aware of environmental issues, and the impact of waste water discharges on dissolved oxygen concentrations in water bodies has started gaining attention [1--4]. The importance of receiving water body quality is also argued in the $E U$ Water Framework Directive $60 / 2000$, which devises a water-quality oriented view of entire watershed systems, and includes new sustainable approaches for disposal of storm-water [5].

Often, water quality models are implemented in order to quantify biological, physical, and chemical transformation of constituents of interest, and to further investigate the impact of altered boundary conditions on aquatic ecosystems [6]. The stream water quality model of water quality assessment problems often incorporates numerical methods to solve the equations. The governing equation of the uniform flow model is a one-dimensional AdvectionDispersion-Reaction equation. In our paper, a mathematical model for the dispersion of pollutants in a river is presented; the Perturbation Iteration Algorithm for assessment of the chemical oxygen demand $(C O D)$ concentration in a river is considered. Pochai et al. [7] had addressed a mathematical model of water pollution by employing the finite element method. The same author(s) [8] 9] implemented the finite difference method $(F D M)$ to the hydrodynamic model with constant coefficients in the uniform reservoir and stream. This model needs the calculation of the substance dispersion given water velocity in the channel to work properly. Padma et al. [10] dealt with mathematical model of water pollution using the Homotopy Analysis Method. Furthermore, Hariharan [11] used another kind of Chebyshev wavelets based approximation method to solve water quality assessment model problem.

During the course of this paper, the new Perturbation Iteration Algorithm introduced by Aksoy et al. [12], to try and find approximate solutions that may help in solving differential equations that stem from problems of assessment of the chemical oxygen demand $(C O D)$ concentration in a river has been considered. An algebraic system is created by applying this method to differential equations.

The remaining structure of this study is as follow. Problem statement is given in section 2, later in this work. A new Perturbation Iteration Algorithm will be reviewed in Section 3. Section 4 discusses the general form of problems in assessment of the chemical oxygen demand $(C O D)$ concentration in a river. To present a clear overview of the procedure, two examples have been presented. Further on, the paper is concluded in Section 5. 


\section{MATHEMATICAL MODEL OF DISPERSION IN A UNIFORM CHANNEL}

The dispersion of chemical oxygen demand $(C O D)$ is described by convection-diffusion equation $(C D E)$ in the domain $[a, b]$

$$
-D_{x} \frac{d^{2} C(x)}{d x^{2}}+U \frac{d C(x)}{d x}+R C(x)-Q=0
$$

where $C(x)$ is the concentration of $C O D$ at point $x \in[a, b] . U$ represents the flow of velocity in x-directions, $D_{x}$ is the diffusivity, $R$ is the substrate decay rate, $Q$ is an increasing rate substrate concentration due to source. The boundary conditions are $C=C$ 。 at $x=a$ and $\frac{d C}{d x}=T_{\circ}$ at $x=b$.

\section{PERTURBATION ITERATION ALGORITHM (PIA)}

Recently, a new iterative method called "Perturbation Iteration Algorithm" has been devised by [12] which proves to be different from all previously published iteration perturbation methods. This new method employs an ingenious combination of perturbation expansions and Taylor series expansions to produce an iteration scheme. Aksoy \& Pakdemirli [12] introduced one correction term in the perturbation expansion and correction terms of only first derivatives in the Taylor series expansion, i.e. $n=1, m=1$. The algorithm is named $P I A(1,1)$. Consider a general second order differential equation,

$$
G\left(u^{\prime \prime}, u^{\prime}, u, \epsilon\right)=0
$$

with $u=u(x)$ and $\epsilon$ the perturbation parameter. Only one correction term is taken in the perturbation expansion.

$$
u_{n+1}=u_{n}+\epsilon\left(u_{c}\right)_{n}
$$

Upon substitution of Eq. (3) into Eq. 23 and expanding in a Taylor series with first derivatives only yields

$$
\begin{aligned}
& G\left(u_{n}^{\prime \prime}, u_{n}^{\prime}, u_{n}, 0\right)+G_{u}\left(u_{n}^{\prime \prime}, u_{n}^{\prime}, u_{n}, 0\right) \epsilon\left(u_{c}\right)_{n}+ \\
& G_{u^{\prime}}\left(u_{n}^{\prime \prime}, u_{n}^{\prime}, u_{n}, 0\right) \epsilon\left(u_{c}^{\prime}\right)_{n}+G_{u^{\prime \prime}}\left(u_{n}^{\prime \prime}, u_{n}^{\prime}, u_{n}, 0\right) \epsilon\left(u_{c}^{\prime \prime}\right)_{n}+ \\
& G_{\epsilon}\left(u_{n}^{\prime \prime}, u_{n}^{\prime}, u_{n}, 0\right) \epsilon=0
\end{aligned}
$$

where $G_{u}=\frac{\partial G}{\partial u}, G_{u^{\prime}}=\frac{\partial G}{\partial u^{\prime}}, G_{u^{\prime \prime}}=\frac{\partial G}{\partial u^{\prime \prime}}, G_{\epsilon}=\frac{\partial G}{\partial \epsilon}$. Reorganizing the equation, one can easily get

$$
\left(u_{c}^{\prime \prime}\right)_{n}+\frac{G_{u^{\prime}}}{G_{u^{\prime \prime}}}\left(u_{c}^{\prime}\right)_{n}+\frac{G_{u}}{G_{u^{\prime \prime}}}\left(u_{c}\right)_{n}=-\frac{G_{\epsilon}+\frac{G}{\epsilon}}{G_{u^{\prime \prime}}}
$$

It is clearly observed that all derivatives are evaluated at $\epsilon=0$, that the above equation is a variable coefficient, and a linear second-order differential equation. Beginning with an initial guess $u_{10}$, first $\left(u_{c}\right)_{\circ}$ is calculated from Eq. 5 5 and then substituted into Eq. (2) to calculate $u_{11}$. This iteration procedure is repeated using Eq. (5) and Eq. (3) until an acceptable result is obtained.

It is noteworthy that if this algorithm is to be used more generally, $n$ correction terms instead of one can be taken in expansion Eq. 3 which would then become a $\operatorname{PIA}(n, m)$ algorithm. The algorithm in question can also be moulded to suit a differential equation system having arbitrary order of derivatives. In this work, however, a case $n=m=1$ has been taken into consideration for simplicity's sake, as more algebra is involved in the construction of iteration solutions for $P I A(1,2)$ and $P I A(1,3)$ as compared to $P I A(1,1)$. Note that all numerical results have been calculated with the help of well-known symbolic software "Mathematica 9.0"

\section{APPLICATION OF PERTURBATION ITERATION ALGORITHM}

So as to show the effectiveness of Perturbation Iteration Algorithm in assessment of water quality model, some numerical examples are presented.

\subsection{Example 1}

Consider the Advection-Dispersion-Reaction Eq. 11 in the form: [8]

$$
C^{\prime \prime}=P(x) C^{\prime}+q(x) C+r(x)
$$

Suppose that there is a plant which discharges waste water into the channel at the starting point $0.0 \mathrm{~km}$ and the $C O D$ concentrations of the waste water are taken to be $1.25 \mathrm{~kg} / \mathrm{m}^{3}$. Let the physical parameter values be diffusion coefficient 2 , flow velocity $u(x)=5-x \mathrm{~m} / \mathrm{s}$, where $x \in[0,2]$, substance decay rate $3 \mathrm{~s}^{-1}$, and rate of change of substance concentration due to the source $1 \mathrm{~kg} / \mathrm{m}^{3} \mathrm{~s}$; so the equation with variable coefficients is obtained as

$$
\begin{gathered}
p(x)=\frac{5-x}{D_{x}}=\frac{5-x}{2} \\
q(x)=\frac{R}{D_{x}}=\frac{3}{2} \\
r(x)=-\frac{Q}{D_{x}}=-\frac{1}{2}
\end{gathered}
$$

By substituting all these values, Eq.(6) becomes

$$
C^{\prime \prime}=\frac{5-x}{2} C^{\prime}+\frac{3}{2} C-\frac{1}{2}
$$

subject to boundary conditions, $C(0)=1.25 ; C^{\prime}(0)=0$. Eq.77 can be written in the following form

$$
2 C^{\prime \prime}-5 C^{\prime}-3 C+\epsilon x C^{\prime}=0
$$

where primes denote differentiation with respect to $x$ and $\epsilon$ is a artificially introduced perturbation parameter. Apply $P I A(1,1)$, Eq. (8) reduces to

$$
2 C_{c}^{\prime \prime}-5 C_{c}^{\prime}-3 C_{c}=\frac{-2 C^{\prime \prime}+5 C^{\prime}+3 C-1}{\epsilon}-x C^{\prime}
$$

Starting with initial trial function and using the formula, the approximate solution at each step are

$$
C_{10}=\frac{5}{4}+\frac{5}{4} x-\frac{5}{16} x^{2}
$$

$$
\begin{aligned}
C_{11}= & -\frac{1}{216\left(1+6 e^{7}\right)}\left(388-120 e^{\frac{2-x}{2}}-3948 e^{\frac{14-x}{2}}-658 e^{3 x}\right. \\
& \left.+120 e^{1+3 x}-240 x+45 x^{2}+6 e^{7}\left(388-240 x+45 x^{2}\right)\right)
\end{aligned}
$$

$$
\begin{aligned}
C_{12}= & -\frac{e^{-\frac{x}{2}}}{1176\left(1+6 e^{7}\right)^{2}}\left(4704 e^{\frac{14+x}{2}}+14112 e^{\frac{28+x}{2}}+392 e^{\frac{x}{2}}-\right. \\
& 33 x e^{7}(4+7 x)-198 e^{14}\left(-196+4 x+7 x^{2}\right)-66 e^{\frac{14+7 x}{2}}(- \\
& \left.\left.196-12 x+21 x^{2}\right)-11 e^{\frac{7 x}{2}}\left(-98-12 x+21 x^{2}\right)\right)
\end{aligned}
$$




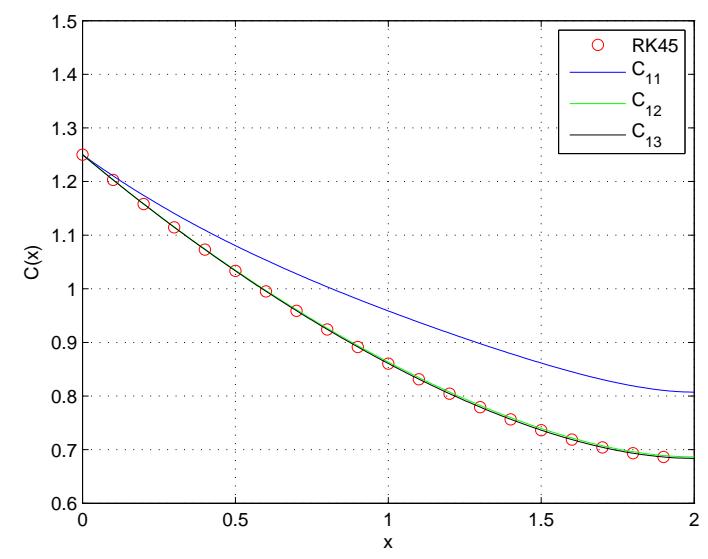

Fig. 1. Graphical comparison of solutions obtained by Perturbation Iteration Algorithm with $R K F 45$ of Example 1

$$
\begin{aligned}
C_{13}= & -\frac{e^{-\frac{x}{2}}}{3226944\left(1+6 e^{7}\right)^{3}}\left(19361664 e^{\frac{14+x}{2}}+\right. \\
& 116169984 e^{\frac{28+x}{2}}+232339968 e^{\frac{42+x}{2}}+ \\
& 10756848 e^{\frac{x}{2}}+1188 e^{21}(537824-12000 x- \\
& \left.21000 x^{2}-1176 x^{3}+343 x^{4}\right)+396 e^{14}(135128- \\
& \left.6512 x-11396 x^{2}-1176 x^{3}+343 x^{4}\right)+ \\
& 33 e^{7}\left(270256-1024 x-17926 x^{2}-1176 x^{3}+\right. \\
& \left.343 x^{4}\right)+792 e^{\frac{28+7 x}{2}}\left(335804+32064 x-56112 x^{2}-\right. \\
& \left.3136 x^{3}+3087 x^{4}\right)+264 e^{\frac{14+7 x}{2}}(167902+23832 x- \\
& \left.41706 x^{2}-3136 x^{3}+3087 x^{4}\right)+22 e^{\frac{7 x}{2}}(134456+ \\
& \left.\left.15600 x-27300 x^{2}-3136 x^{3}+3087 x^{4}\right)\right)
\end{aligned}
$$

To make sure of the accuracy of the Perturbation Iteration Algorithm $(P I A)$ solution, a comparison between the solutions obtained by $P I A$ and numerical method was made and is displayed in Table 1. Graphical representation of the solution using PIA and Runge-Kutta-Fehlberg fourth fifth order method is visible in Figure 1 ; an excellent agreement can be observed.

\subsection{Example 2}

Consider the Convection-Diffusion problem [13]

$$
-2\left(2 x^{2}+\tan x^{2}\right) u(x)=u^{\prime \prime}(x) ; \quad x \in[0,1]
$$

with boundary condition, $u(0)=1, u^{\prime}(0)=0$. The exact solution is $u(x)=\cos x^{2}$, where prime denote differentiation with respect to $x$. By introducing artificially small perturbation parameter $\epsilon$, Eq.(14) can be written as

$$
-2\left(2 \epsilon x^{2}+\tan \left(\epsilon x^{2}\right)\right) u(x)=u^{\prime \prime}(x)
$$

Table 1. Values of solutions via Perturbation Iteration Algorithm and numerical method $R K F 45$

\begin{tabular}{ccccc}
\hline$x$ & $R K F 45$ & $C_{11}$ & $C_{12}$ & $C_{13}$ \\
\hline 0.0 & 1.2500 & 1.250000000 & 1.250000000 & 1.250000000 \\
0.1 & 1.2031 & 1.210551205 & 1.203315976 & 1.203148808 \\
0.2 & 1.1580 & 1.174209587 & 1.158457540 & 1.158085577 \\
0.3 & 1.1147 & 1.140631784 & 1.115401624 & 1.114789357 \\
0.4 & 1.0731 & 1.109495168 & 1.074126559 & 1.073243138 \\
0.5 & 1.0333 & 1.080498223 & 1.034613592 & 1.033435016 \\
0.6 & 0.9952 & 1.053361399 & 0.996848684 & 0.995359676 \\
0.7 & 0.9588 & 1.027828581 & 0.960824692 & 0.959020263 \\
0.8 & 0.9242 & 1.003669375 & 0.926544033 & 0.924430702 \\
0.9 & 0.8914 & 0.980682480 & 0.894021941 & 0.891618536 \\
1.0 & 0.8604 & 0.958700520 & 0.863290462 & 0.860628369 \\
1.1 & 0.8313 & 0.937596824 & 0.834403328 & 0.831526002 \\
1.2 & 0.8042 & 0.917294820 & 0.807441853 & 0.804403323 \\
1.3 & 0.7792 & 0.897780963 & 0.782521991 & 0.779384044 \\
1.4 & 0.7564 & 0.879122407 & 0.759802619 & 0.756630338 \\
1.5 & 0.7362 & 0.861491065 & 0.739495026 & 0.736350442 \\
1.6 & 0.7187 & 0.845196300 & 0.721873374 & 0.718807268 \\
1.7 & 0.7042 & 0.830729244 & 0.707285566 & 0.704328117 \\
1.8 & 0.6932 & 0.818822803 & 0.696163396 & 0.693315676 \\
1.9 & 0.6862 & 0.810532836 & 0.689029963 & 0.686260701 \\
\hline
\end{tabular}

Applying Perturbation Iteration Algorithm on Eq.15, it becomes

$$
u_{c}^{\prime \prime}=\frac{u^{\prime \prime}}{\epsilon}-6 x^{2} u
$$

Choosing the initial guess $u_{10}=1$. Using the iterative formula, the successive solutions are:

$$
\begin{gathered}
u_{11}=1-\frac{1}{2} x^{4} \\
u_{12}=1-\frac{1}{2} x^{4}+\frac{3}{56} x^{8} \\
u_{13}=1-\frac{1}{2} x^{4}+\frac{3}{56} x^{8}-\frac{3}{1232} x^{12} \\
u_{14}=1-\frac{1}{2} x^{4}+\frac{3}{56} x^{8}-\frac{3}{1232} x^{12}+\frac{3}{49280} x^{16}
\end{gathered}
$$

In Table 2, the comparison between the exact solution with the successive approximation of solution obtained by new Perturbation Iteration has been made. Figure 2 graphically endorsed this statement. Perturbation Iteration Algorithm provide more realistic series solutions that converge very rapidly, usually only a few of its iterations leading to very accurate result

\section{CONCLUDING REMARKS}

In this paper, the solution of the one dimensional steady AdvectionDiffusion-Reaction equation with variable inputs using Perturbation Iteration approach and Runge-Kutta-Fehlberg fourth-fith order method has been presented. Both approximate analytical and numerical results are obtained for the given problems. The validity of the proposed procedure, called the Perturbation Iteration Algorithm $(P I A)$, was demonstrated through two examples, and an exceptional agreement was found between the approximate analytic 


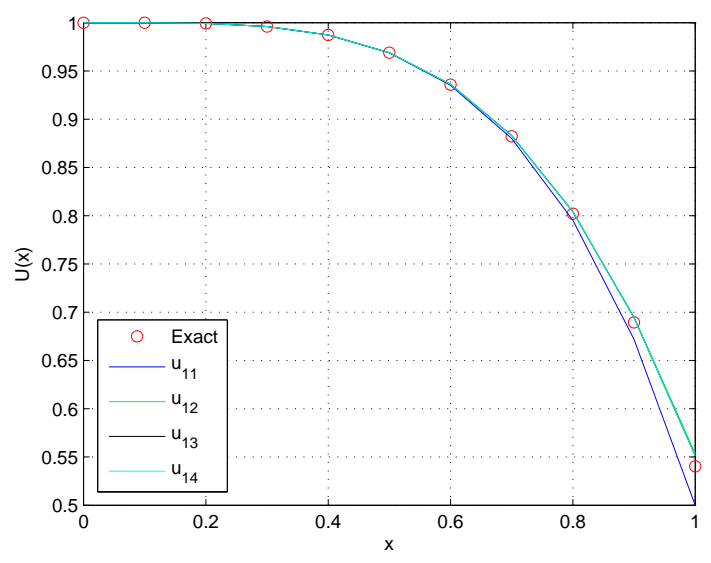

Fig. 2. Graphical comparison of solutions obtained by $P I A(1,1)$ with exact solution of Example 2

Table 2. Values obtained by Perturbation Iteration approach and Exact solution (Example 2)

\begin{tabular}{cccccc}
\hline$x$ & Exact & $u_{11}$ & $u_{12}$ & $u_{13}$ & $u_{14}$ \\
\hline 0.0 & 1.000000 & 1.000000 & 1.000000 & 1.000000 & 1.000000 \\
0.1 & 0.999950 & 0.999950 & 0.999950 & 0.999950 & 0.999950 \\
0.2 & 0.999200 & 0.999200 & 0.999200 & 0.999200 & 0.999200 \\
0.3 & 0.995953 & 0.995950 & 0.995954 & 0.995954 & 0.995954 \\
0.4 & 0.987227 & 0.987200 & 0.987235 & 0.987235 & 0.987235 \\
0.5 & 0.968912 & 0.968750 & 0.968959 & 0.968959 & 0.968959 \\
0.6 & 0.935897 & 0.935200 & 0.936100 & 0.936094 & 0.936095 \\
0.7 & 0.882333 & 0.879950 & 0.883038 & 0.883005 & 0.883005 \\
0.8 & 0.802096 & 0.795200 & 0.804188 & 0.804020 & 0.804022 \\
0.9 & 0.689498 & 0.671950 & 0.695011 & 0.694323 & 0.694334 \\
1.0 & 0.540302 & 0.500000 & 0.553571 & 0.551136 & 0.551197
\end{tabular}

results and numerical simulation results. The proposed scheme provides us with a straightforward and precise way to optimally control and adjust the convergence of a solution; it can give very good approximations in a few terms.

\section{CONFLICT OF INTERESTS}

The authors declare that there is no conflict of interests regarding the publication of this paper.

\section{ACKNOWLEDGMENT}

We thank the reviewers for their thorough efforts in editing our paper and highly appreciate the comments and constructive criticism that significantly contributed in improving the quality of the publication. The authors also thank Ms.Wishaal Khalid for proofreading our research paper.

\section{REFERENCES}

[1] Refsgaard, J.C., Van der Sluijs, J.P., Hojberg, A.L. \& Vanrolleghem, P.A. (2007) Uncertainty in the environmental modelling process-a framework and guidance. Environmental Modelling \& Software 22. pp 1543-1556
[2] Dubus, Igor.G., Brown, C.D. \& Beulke, S. 2003 Sources of uncertainty in pesticide fate modeling. The Science of the Total Environment 317(1-3). pp 53-72

[3] Harremoes, P. (1988) Stocastic models for estimation of extreme pollution from urban runoff. Water Resources, 22(8). pp 1017-1026

[4] Beck, M.B. (1987) Water quality modelling: a review of the analysis of uncertainty. Water Resources Research, 23. pp 1393-1442

[5] Chave, P. (2001) The EU Water Framework Directive: An Introduction. IWA Publishing, London.

[6] Wagenschein, D. \& Rode, M. (2008) Modelling the impact of river morphology on nitrogen retention - a case study of the Weisse Elster River (Germany), Ecological Modelling, 211. 224-232

[7] Pochai, N. \& Tangmanee, S. (2007) A mathematical model of water pollution using finite element method, Contributions in Mathematics and Applications, East-West J. Math. Spec., pp 143-154

[8] Pochai, N. \& Depana, R. (2011) An optimal control of water pollution in a stream using a finite difference method, World Academy of Science, Engineering and Technology, 6(56). pp 1186-1188

[9] Pochai, N. (2009) A numerical computation of the nondimensional form of a non-linear hydrodynamic model in a uniform reservoir, Nonlinear Analysis: Hybrid Systems, 3(4). pp 463-466

[10] Padma, S., Hariharan, G., Kannan, K. \& Srikanth, R. (2013) Homotopy Analysis Method to Water Quality Model in a Uniform Channel, Applied Mathematical Sciences, 7(22). pp 1057-1066

[11] Hariharan, G. (2014) An efficient wavelet based approximation method to water quality assessment model in a uniform channel, Ain Shams Engineering Journal, 5. pp 525-532

[12] Aksoy, Y. \& Pakdemirli, M. (2010) New perturbationiteration solutions for Bratu-type equations, Computers and Mathematics with Applications, 59(8). pp 2802-2808

[13] Hariharam, G. \& Kannan, K. (2010) A comparative study of Haar Wavelet method and restrictive Taylor's series method for solving convection-duffusion equation, International Journal for Computational method in Engineering Science and Mechanics, 11(4). pp 173-184 